\title{
PROSPECÇÃO INTERACTIVA DE ENDOCARSO ATRAVÉS DE UMA APLICAÇÃO SIG
}

\author{
Hugo Salinas Vargas ${ }^{1}$ \\ José António Almeida \\ José Carlos Kullberg \\ Maria da Graça Brito
}

\begin{abstract}
Resumo - Os parâmetros que determinam o desenvolvimento de erosão cársica em profundidade - endocarso - são complexos e difíceis de avaliar, devido, por um lado, à diversidade dos factores condicionantes e, por outro, à dificuldade em quantificar a sua interacção e grau de influência efectivos. Neste trabalho é apresentada uma metodologia em ambiente SIG, desenvolvida com o objectivo de identificar as áreas mais propícias à ocorrência de endocarso. O estudo incidiu sobre uma área piloto a Oeste de Sesimbra, onde estão identificadas várias dessas estruturas, com particular relevância para as grutas do Zambujal e do Frade. Um dos principais objectivos é integrar o conjunto de parâmetros que, de algum modo, controlam o desenvolvimento deste tipo de carsificação numa aplicação interactiva, de forma a testar, expeditamente e com avaliação pericial, a importância relativa dos parâmetros intervenientes.
\end{abstract}

Palavras-chave: Erosão cársica, endocarso, classificação pericial, carta de aptidão.

Abstract - GIS aPPLication For interactive prospection of EndoKarst. The parameters that affect the development of karst erosion at depth - endokarst - are complex and difficult to understand, due to both their diversity and the difficulty of quantifying the actual contribution of each of them. This work presents a methodology developed in a GIS environment with the aim of identifying the areas in which endokarst is most likely to occur. This case study was conducted within a sample area located to the west of Sesimbra where several of these structures have been identified, with a special emphasis on the caves of Zambujal and Frade. The suggested model is based on the interactive combinatory analysis of the dependent variables according to skillful criteria, taking into account their relative contributions to the development of endokarst. One of the main goals of this study is the development of an interactive software application enabling to test and evaluate the relative importance of the selected parameters. A decision was made to use a simple classification of the supervised or skillful type, in which the variables, their inter-relations and their relations with the surrounding areas are conveyed under the summarised form of a ranking of their relative relevance, as measured by their

1 Investigador do Centro de Investigação em Geociências Aplicadas (CIGA), FCT/UNL, Monte de Caparica, 2829-516 Caparica, Portugal. Tel/Fax: +351 212948 349. E-mail: hugo@ciga.fct.unl.pt 
expected contribution to karstification. The output of the model consists of a set of maps that indicate the likelihood of the occurrence of endokarst at any given point. The hierarchical relevance of each variable, the selection of larger or smaller buffer areas for fractures and fracture intersections, the choice of appropriate variables and their subdivision into a number of categories allow for scrutiny to be applied at a series of different levels, rendering possible the skillful calibration of the results to all the places where the predominant geology is of the carbonate type. At the same time, the comparative analysis of these results provides an excellent opportunity for us to understand the parameters that most contribute to the formation of endokarst. The suggested model was tested interactively and the latter analysis and validation of the results allowed for the positive identification of the appropriate sequence of those variables that most affect the formation of endokarst.

Key words: Karst erosion, endokarst, aptitude map, skillful classification.

Resumé - Prospection INTERACTIVE DE L'ENDOKARST À TRAVERS D'UN SIG. Les paramètres qui conditionnent le développement de l'érosion karstique en profondeur - endokarst - sont complexes et difficiles d'évaluer étant données, d'une part la diversité des éléments engagés et, d'autre part, la difficulté de quantifier l'interaction et le réel degré d'influence de ces mêmes éléments. Ce travail présente une méthodologie dans le cadre SIG qui a comme objectif l'identification de secteurs de terrain les plus favorables à l'occurrence d'un endokarst. L'étude a été faite dans un terrain pilote à l'Ouest de Sesimbra, où ont été identifiées plusieurs structures de ce type et où se situent les grottes de Zambujal et de Frade. Le prototype de modèle qu'on propose s'appuie dans l'analyse combinatoire des facteurs ordonnés selon des critères d'expertise, c'est-à-dire, selon leur importance tenant compte de leur contribution à la formation d'un endokarst. Lorsque que l'on identifie les paramètres intervenants, le résultat du modèle indique le degré d'aptitude à l'occurrence d'un endokarst, pour chaque unité d'espace. Les possibilités d'ordenation des paramètres, la sélection de la distance d'influence concernant la lithologie et l'intersections des fractures, et même le choix des paramètres et leur bifurcation - avec plusieurs possibilités, qui envisagent appliquer la discrimination à des niveaux différents - sont des possibilités d'application du modèle qui permettent le calibrage expertisé des résultats à plusieurs emplacements. Un des objectifs principaux est celui d'intégrer, dans une application interactive, l'ensemble des paramètres que controlent le développment de ce type de karsification; cette application permettrait d'expertiser rapidement l'importance relative des paramètres en question. Une comparaison de ce type est une excellente opportunité pour comprendre les paramètres les plus importants dans la formation de l'endokarst.

Mots-clés: Érosion karstique, endokarst, carte d'aptitude, expertise.

\section{INTRODUÇÃO}

\section{Objectivos}

De uma forma abrangente, a erosão cársica ou carsificação é definida como o conjunto de processos de alteração química e mecânica diferenciais que, sob determinadas condições do meio, actuam sobre corpos rochosos solúveis, como os calcários ou os dolomitos (SweEting, 1972; FetTer, 1980). Estes processos são 
mais eficazes quando actuam em formações calcárias espessas e fracturadas e em climas com valores de temperatura e humidade mais elevados (PARIZEK, 1976; Smith and Atkinson, 1976; Yevjevich, 1980). A alteração ocorrerá tanto à superfície como em profundidade, donde resulta a formação de endocarso. Os processos naturais que levam ao seu desenvolvimento são complexos e difíceis de avaliar, essencialmente por duas razões: por um lado, a diversidade e a variabilidade local destes e, por outro, a sua interacção e quantificação, mesmo que relativa, ou seja, a hierarquização dos processos.

O desenvolvimento de um modelo integrado em SIG com vista à prospecção de carso profundo reside na necessidade de compreender e sistematizar as interacções que os vários processos naturais assumem. Assim, foi desenvolvida uma aplicação em Visual Basic que, em conjunto com o SIG ArcGIS, permitiu (de uma forma expedita e interactiva) testar várias combinatórias, segundo a importância relativa dos factores envolvidos neste processo. Esta aplicação foi testada numa área piloto a Oeste de Sesimbra, onde estão identificadas várias estruturas endocársicas, com particular relevância para as grutas do Zambujal ${ }^{2}$ e do Frade, apresentando assim potencial para a ocorrência de outras cavidades similares (fig. 1).

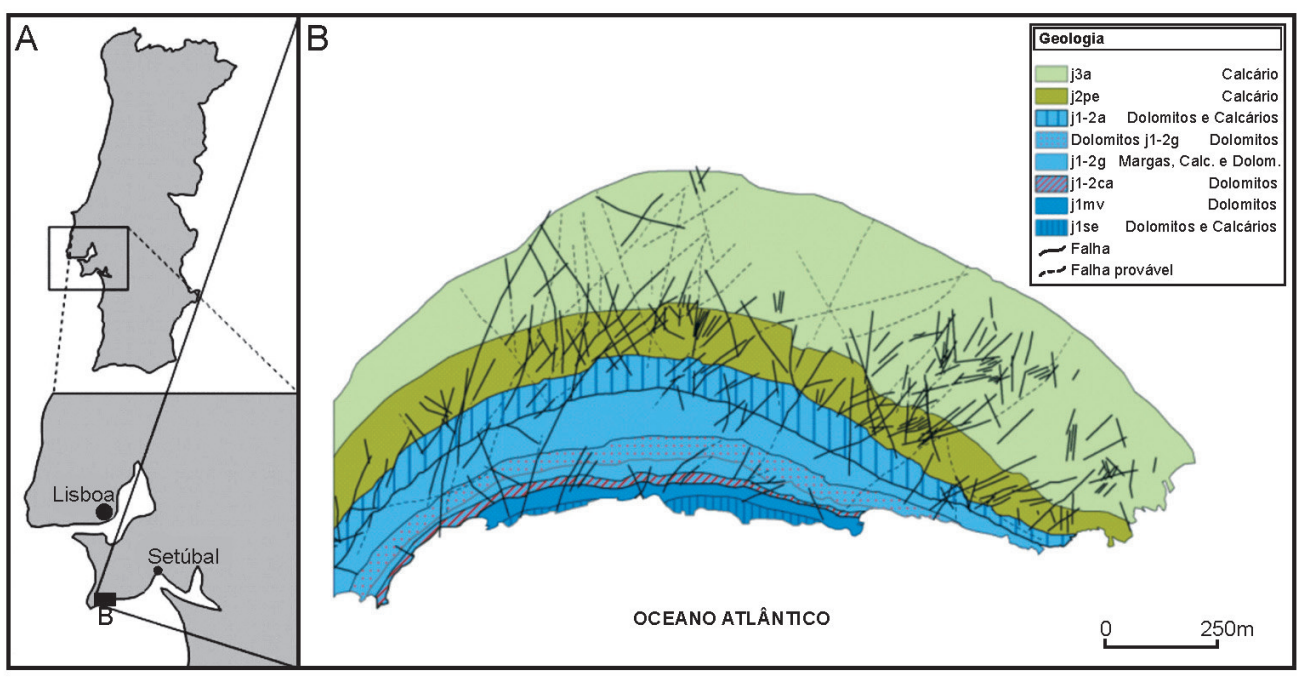

Fig. 1 - a) Localização da área piloto na região da Arrábida e b) Geologia. Unidades litostratigráficas modificadas de MANupPella et al. (1994, 1999). Ver Quadro I.

Fig. 1 - a) Location of the sample area in the Serra da Arrábida and b) Geology. Lithostratigraphic units modified from MANUPPELLA et al. (1994, 1999). See Table I.

${ }^{2}$ Classificada como Sítio de Interesse Espeleológico pelo Decreto-Lei n. ${ }^{\circ}$ 140/79, de 21 de Março. 


\section{Selecção dos principais factores que condicionam a formação de endocarso}

O fenómeno de carsificação pode ser descrito por um modelo conceptual que represente, por descrições ou diagramas, as suas características e o modo como os factores condicionantes controlam o seu desenvolvimento.

Com base em trabalhos de referência sobre a carsificação subterrânea (compilados por BLAIR, 1986), pode afirmar-se que o desenvolvimento deste tipo de morfologia depende da interacção de pelo menos sete factores importantes, a saber: litologia, estrutura, relevo, hidrologia, clima, vegetação e tempo (geológico).

Anteriormente, Ford (1976) efectuou uma análise sistemática, no sentido de quantificar o grau de dissolução de vários tipos de formações carbonatadas em função dos factores hidrológicos e do clima, não chegando, todavia, a estabelecer intervalos quantitativos sobre a influência de cada um. Efectivamente, todos os estudos até à data (Corbel, 1959; CviJic, 1957; Ford, 1976; Roglic, 1972) apontam para o facto de que é extremamente difícil, ou mesmo impossível, quantificar numericamente a relação entre os factores e a propensão à ocorrência de carso, embora se possa, de uma forma qualitativa, emitir uma opinião pericial, válida apenas para as características de cada local.

\section{METODOLOGIA PROPOSTA}

Este estudo compreende as seguintes fases:

a) Recolha e digitalização de toda a informação de partida relevante para a ocorrência de endocarso, nomeadamente de natureza geológica (unidades litológicas e fracturação - carta geológica e levantamentos de campo), modelo digital do terreno e áreas de ocorrência de dissolução superficial (terra rossa);

b) Processamento da informação utilizando funções de análise espacial do ArcGIS: localização dos pontos de intersecção de fracturas, cálculo de áreas de influência relativamente a fracturas e pontos de intersecção de fracturas;

c) Construção de uma carta de declives a partir do modelo digital de terreno;

d) Codificação da informação de partida em classes, de acordo com a maior ou menor influência com que intervêm na formação de endocarso e transformação de toda a informação vectorial para estrutura matricial;

e) Execução do modelo e classificação de toda a área de estudo, em função dos vários factores que propiciam áreas de maior aptidão para o desenvolvimento de endocarso. O modelo foi executado interactivamente de forma a experimentar algumas relações relativamente à ordem de importância dos factores intervenientes, propostas por conhecimento pericial; 
f) Análise dos resultados e validação do modelo. Identificação da sequência (ou sequências) dos factores, simultaneamente validada pelo conhecimento pericial e pela ocorrência efectiva de carso.

Para este estudo, e relativamente a um local com as características da Serra da Arrábida, foram listados previamente todos os factores passíveis de contribuir para a ocorrência de endocarso. Estes factores foram subdivididos em dois conjuntos: primários (relativos ao maciço e suas descontinuidades) e derivados (relativos ao enquadramento climático e temporal). Os factores primários foram ainda agrupados em independentes e dependentes conforme a sua utilização é ou não discriminante a esta escala de trabalho.

O modelo conceptual associado ao fenómeno de carsificação profunda na Serra da Arrábida pode ser representado pelo seguinte diagrama (fig. 2).

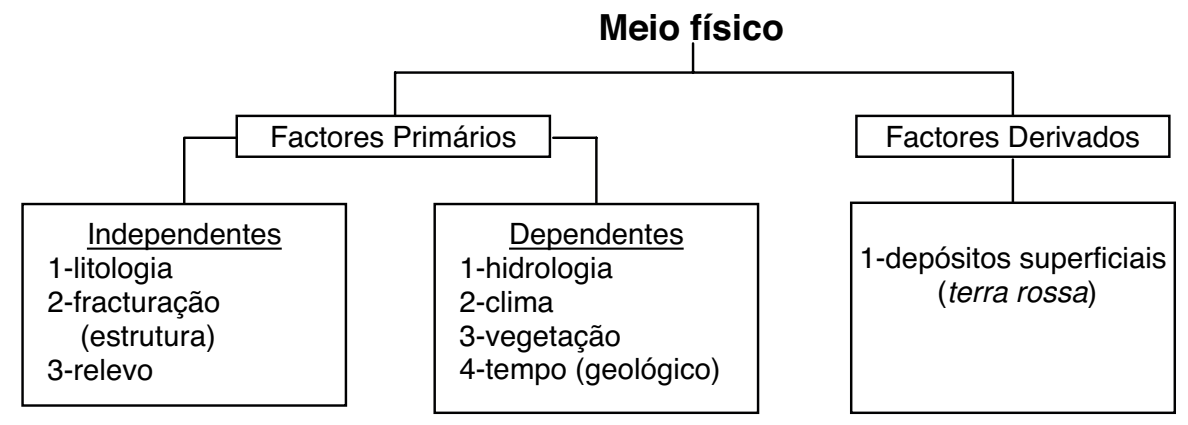

Fig. 2 - Diagrama representativo dos factores a considerar num estudo de aptidão ao desenvolvimento de endocarso.

Fig. 2 - List of the relevant parameters in endokarst prospecting studies.

Neste trabalho foi considerado um único factor extrínseco, que representa a ocorrência de depósitos superficiais do tipo terra rossa. Este factor é extremamente importante, pois denuncia, superficialmente, a existência de fenómenos de alteração preferencial. Ou seja, a ocorrência daqueles depósitos constitui uma evidência de zonas principais de dissolução de carbonatos, agora parcial ou totalmente impermeabilizadas. Adicionalmente, a sua presença dificulta a recolha de dados uma vez que oculta vestígios de outros factores. Justifica-se assim a sua integração para efeitos de prospecção de endocarso.

Dos oito factores (primários e derivados) de intervenção no processo de carsificação (na prática, da fracturação - estrutura - derivam-se dois factores), apenas foram considerados neste trabalho aqueles que são independentes: a litologia, a estrutura (fracturação + densidade de intersecções) e o relevo (carta de declives), para além dos depósitos superficiais. Deve-se este facto à inexistência de informação sobre a hidrologia e à invariância dos restantes factores ditos dependentes na escala de trabalho considerada. 
Com a metodologia proposta, pretende-se atribuir, para cada célula de uma partição do espaço, um grau de aptidão à ocorrência de endocarso, uma vez identificados os vários factores intervenientes segundo várias modalidades ordinais ou nominais. Neste caso particular, é inviável a utilização dos classificadores clássicos da análise de dados, nomeadamente, análise de correspondências, árvores de classificação ou redes neuronais, uma vez que não se conhece (nem há maneira de conhecer) a relação factores/ocorrência de endocarso, dado que não existe uma cartografia de endocarso sistemática em nenhuma das áreas para calibrar um modelo do tipo factores/resposta. Assim, optou-se por proceder a uma classificação supervisionada ou pericial, onde o conhecimento prévio dos factores e das relações entre eles e com o meio no local estudado permitiu que estes fossem ordenados "pericialmente», segundo a sua importância relativa na contribuição do carso. Do mesmo modo, as modalidades de cada factor foram convertidas em nominais $(1, \ldots)$, que exprimem a maior ou menor apetência de cada factor na contribuição para a ocorrência de endocarso. Se $n$ for o número de factores, $(\mathrm{A}, \mathrm{B}, \mathrm{C}, \ldots)$ a lista dos factores ordenados por ordem decrescente de importância relativa e $a_{m}, b_{m}, c_{m}, \ldots$ o número de modalidades de cada factor, cada célula $x_{u}$ é codificada numa sequência do seguinte modo: $\operatorname{COD}\left(x_{u}\right)=\left\{a_{i}, b_{j}\right.$, $\left.c_{k}, \ldots\right\}$, em que $a_{i}$ representa a modalidade do factor A em $x_{u}, b_{j}$ representa a modalidade do factor B em $x_{u}$,..., etc.

Entretanto, se pensarmos em todas as possibilidades combinatórias das modalidades para uma dada sequência ordenada de factores - o número de combinatórias diferentes admissíveis é igual a $\mathrm{M}=a_{m} \cdot b_{m} \cdot c_{m} \ldots-$ verifica-se que a combinatória onde todas as modalidades aparecem com o valor nominal mínimo corresponde à situação de menor aptidão à ocorrência de endocarso e que, pelo contrário, a combinatória onde todas as modalidades aparecem com o valor nominal máximo corresponde à situação de maior aptidão. Para as modalidades intermédias $(\mathrm{M}=2)$, aplica-se uma simples classificação baseada na posição de ordem da amostra na árvore total de combinatórias dos factores/ /modalidades, e sempre baseada no princípio de ordem estabelecido para os factores e modalidades. A título meramente ilustrativo, considere-se a árvore de combinatórias para um exemplo simples, com três factores e $2 \times 3 \times 2$ modalidades (fig. 3).

Observa-se que a qualquer combinatória intermédia corresponde um grau de aptidão que é condicionado hierarquicamente aos factores pela ordem em que são colocados. Por exemplo, a combinação $(1,3,1)$ corresponde à posição de ordem 5. Todas as posições de ordem menores do que 5 correspondem a situações em que, pelo menos, uma das modalidades de um factor mais importante tem um valor inferior. Pelo contrário, todas as posições de ordem superior a 5 correspondem a situações em que, pelo menos, uma das modalidades de um factor mais importante tem um valor superior. 


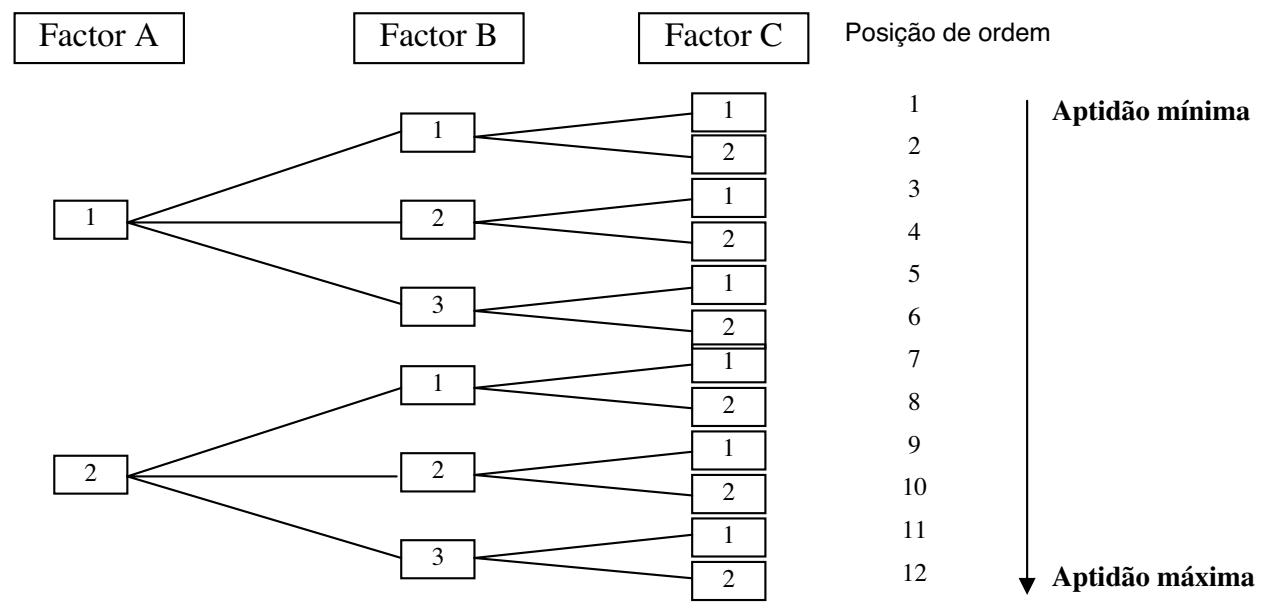

Fig. 3 - Exemplo de combinatórias para três factores e $2 \times 3 \times 2$ modalidades.

Fig. 3 - Set of combinations in a simple example of three variables and 2 by 3 by 2 classes.

\section{TRATAMENTO DA INFORMAÇÃO GEOGRÁFICA E ALFANUMÉRICA}

\section{Recolha e digitalização da informação utilizada}

Esta etapa consistiu na preparação e transformação para formato digital da informação relevante relativa aos factores que influenciam a carsificação, nomeadamente:

a) recolha de informação publicada relativa à litologia e estrutura do maciço em estudo;

b) digitalização, georreferenciação das fotografias aéreas e foto-interpretação geológica, para a delimitação das diferentes unidades litostatigráficas e identificação das estruturas existentes (falhas e fracturas);

c) cartografia geológica de campo à escala 1:5 000, com auxílio de um aparelho GPS (Global Positioning System) e do sistema portátil ArcPAD;

d) incorporação da altimetria e ortofotomapa em formato digital;

e) levantamento e incorporação das áreas de ocorrência de dissolução superficial - terra rossa.

O quadro seguinte resume os temas de partida, descrição das classes e estrutura da informação. 
Quadro I - Temas cartográficos de partida.

Table I - List of used coverages.

\begin{tabular}{|c|c|c|c|}
\hline Tema & \multicolumn{2}{|l|}{ Descrição Classes } & Estrutura da informação \\
\hline Litologia & $\begin{array}{l}\text { Calcários (J2pe, J3a) } \\
\text { Dolomitos e calcários (J1-2a, J1se) } \\
\text { Dolomitos (J1-2g, J1-2Ca, J1mv) } \\
\text { Margas, calcários e dolomitos (J1-2g) }\end{array}$ & $\begin{array}{l}-4 \\
-3 \\
-2 \\
-1\end{array}$ & Vectorial, polígonos \\
\hline Fracturação & $\begin{array}{l}\text { Falhas prováveis } \\
\text { Falhas provadas }\end{array}$ & & Vectorial, linhas \\
\hline Modelo digital terreno & Cotas & & Matricial \\
\hline $\begin{array}{l}\text { Áreas de dissolução } \\
\text { superficial preferencial }\end{array}$ & $\begin{array}{l}\text { Evidenciando dissolução preferencial } \\
\text { Sem evidências de dissolução preferenci }\end{array}$ & & Vectorial, polígonos \\
\hline
\end{tabular}

\section{Pré-processamento da informação de partida}

Esta etapa de processamento preliminar consistiu na construção de informação derivada que evidencia espacialmente a influência das estruturas geológicas identificadas, utilizando funções de análise espacial do ArcGis.

Relativamente à fracturação, efectuaram-se os passos ilustrados pela figura 4, sobre dados em estrutura vectorial:

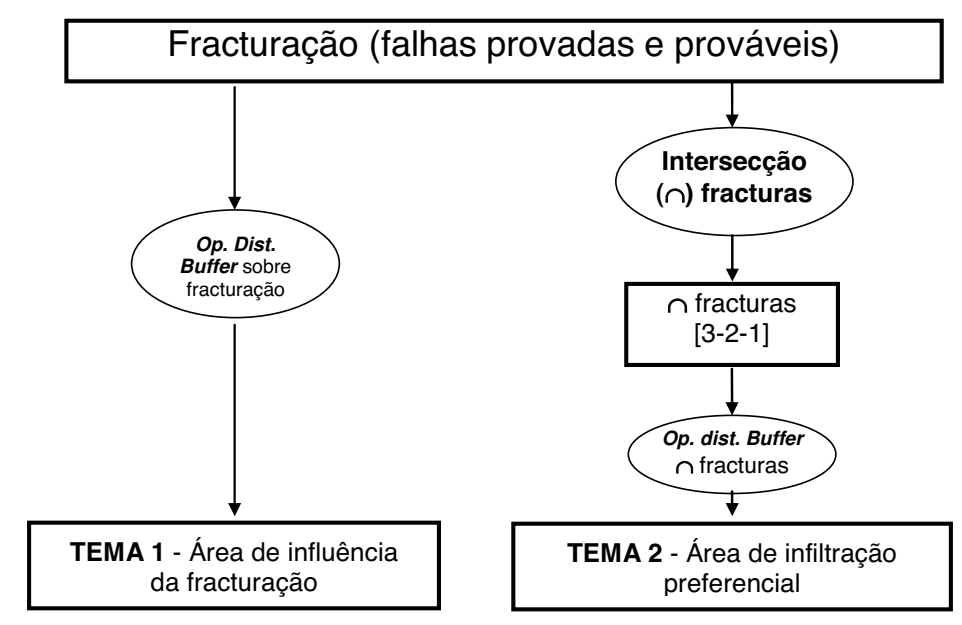

Fig. 4 - Esquema ilustrativo do pré-processamento do tema fracturação.

Fig. 4 -Diagram showing pre-processing steps for the fracture coverage.

a) Identificação da área de influência das fracturas utilizando o operador de distância buffer sobre a cobertura da fracturação de uma forma diferencial: 20 metros para falhas provadas e 15 metros para falhas prováveis. Todos os polígonos resultantes foram dissolvidos (fig. 5a). 


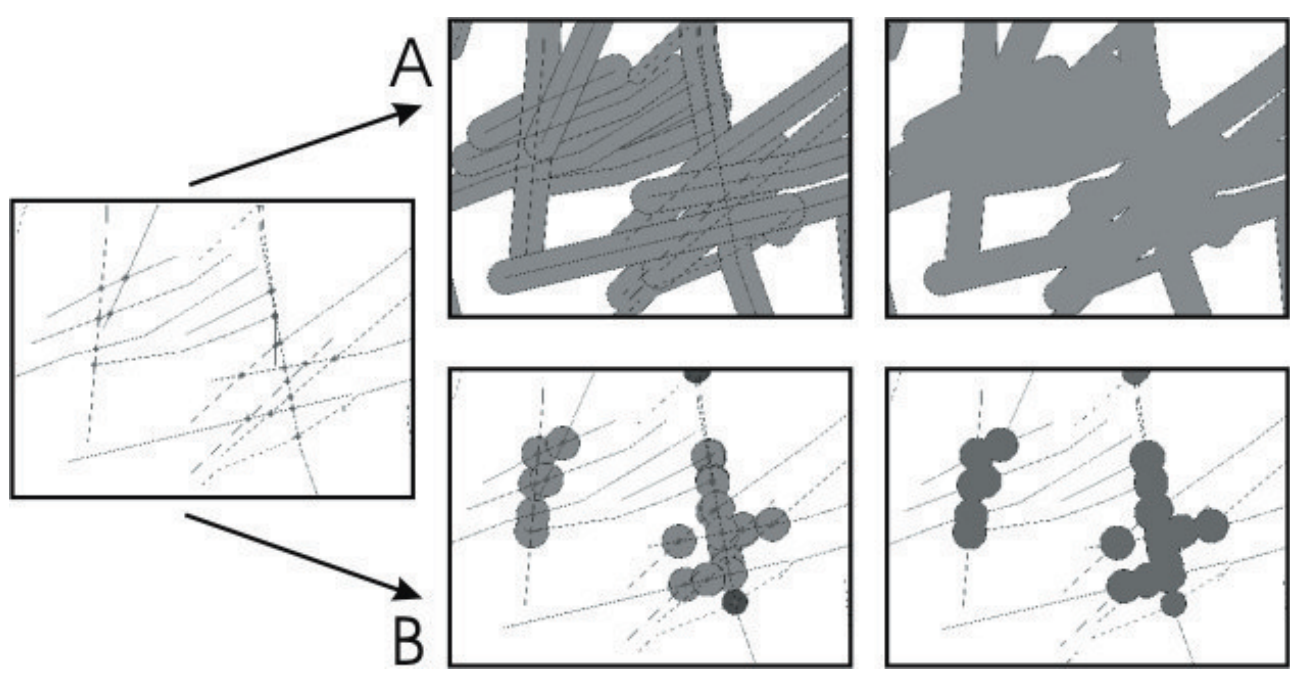

Fig. 5 - a) Área de influência da fracturação e b) zonas de infiltração preferencial resultantes das intersecções de fracturas.

Fig. 5 - a) Influence areas for fracturation and b) preferential infiltration areas resulting from intersection of fractures.

b) Localização dos pontos de intersecção de fracturas e classificação automática de cada ponto com os códigos $[1 ; 2 ; 3]$ consoante a intersecção seja resultante de: 1) duas fracturas provadas; 2 ) uma fractura provada e uma provável e 3) duas fracturas prováveis. Identificação da área de influência dos pontos de intersecção das fracturas, utilizando o operador de distância buffer de uma forma diferencial: código 1 - 20 metros; código 2 - 15 metros e código 3 - 10 metros. Novamente, todos os polígonos resultantes foram dissolvidos (fig. 5b).

Todos os valores para os parâmetros intervenientes nesta análise resultaram do conhecimento pericial do fenómeno aplicado à região, como por exemplo a escolha de 20 e 15 metros, respectivamente, para a área de influência lateral de fracturas provadas e prováveis.

\section{Classificação da informação de partida}

Nesta etapa procedeu-se à categorização e codificação numérica dos parâmetros descritores do sistema, que traduzem a maior ou menor aptidão ao desenvolvimento de endocarso. Aos valores mais altos do índice está associado um grau de aptidão elevado e aos valores mais baixos um grau de aptidão baixo (Quadro II). 
Quadro II - Factores condicionantes da aptidão ao desenvolvimento de endocarso e suas classes.

Table II - Main variables affecting the development of endokarst.

\begin{tabular}{|c|c|c|c|}
\hline \multicolumn{2}{|c|}{ Factores } & \multirow{2}{*}{\begin{tabular}{l}
\multicolumn{1}{c}{ Classes } \\
4 - elevado \\
3 - médio \\
2 - médio baixo \\
1 - quase nulo
\end{tabular}} & \multirow{2}{*}{$\begin{array}{c}\text { Observações } \\
\text { Grau de aptidão à dissolução }\end{array}$} \\
\hline \multirow{4}{*}{$\begin{array}{l}\text { Primários } \\
\text { independentes }\end{array}$} & Litologia & & \\
\hline & Fracturação & $\begin{array}{l}2 \text { - dentro } \\
1 \text { - fora }\end{array}$ & $\begin{array}{l}\text { Fora ou dentro da área de influência } \\
\text { da fracturação }\end{array}$ \\
\hline & Intersecções & $\begin{array}{l}2 \text { - dentro } \\
1 \text { - fora }\end{array}$ & $\begin{array}{l}\text { Fora ou dentro da área de influência } \\
\text { de intersecções de fracturação } \\
\text { - estabelecimento de canais prefe- } \\
\text { renciais de infiltração }\end{array}$ \\
\hline & Declive & $\begin{array}{l}3 \text { - elevado }\left(0^{\circ} \text { a } 6^{\circ}\right) \\
2-\text { médio }\left(6^{\circ} \text { a } 25^{\circ}\right) \\
1-\text { baixo }\left(>25^{\circ}\right)\end{array}$ & $\begin{array}{l}\text { Maior ou menor potencial de infil- } \\
\text { tração consoante o declive }\end{array}$ \\
\hline Derivados & Zonas de dissolução & $\begin{array}{l}2-\text { presença } \\
1-\text { ausência }\end{array}$ & $\begin{array}{l}\text { Depósitos superficiais resultantes } \\
\text { da dissolução dos carbonatos }\end{array}$ \\
\hline
\end{tabular}

\section{APLICAÇÃO DO MODELO}

\section{Aplicação do modelo de classificação à aptidão ao desenvolvimento de endocarso}

Nesta fase foi executado o modelo de análise das combinatórias existentes, atribuindo a cada célula um grau de aptidão relativo, segundo determinada sequência de importância atribuída aos factores analisados e às modalidades existentes. O resultado final é um novo tema matricial, onde aos valores mais elevados correspondem zonas de maior aptidão ao desenvolvimento 'actual' de endocarso.

Neste modelo é importante realçar a intervenção da litologia, que se encontra codificada em 4 classes ( 1 a 4, com graus de aptidão à dissolução, variando de quase nulo a elevado). Nesta situação, em que um tema apresenta várias modalidades e em que o nível discriminante de cada modalidade não tem a mesma importância relativa, é preferível que a sua intervenção discriminante seja feita a dois níveis: em primeiro lugar a separação entre a modalidade designada por quase nula do conjunto das restantes e, posteriormente, as restantes modalidades (médio baixo, médio e elevado). Com esta subdivisão, consegue-se que a litologia tenha um efeito discriminante com dois pesos (eliminando a priori, áreas com aptidão nula ou quase nula das restantes).

Para testar o modelo, foram efectuadas várias combinatórias da importância relativa dos parâmetros. Comparando os resultados com alguns locais onde 
hoje se reconhece a existência de fendas ou grutas, duas combinatórias de ordem dos factores merecem um destaque particular (fig. 6a e 6b, respectivamente).
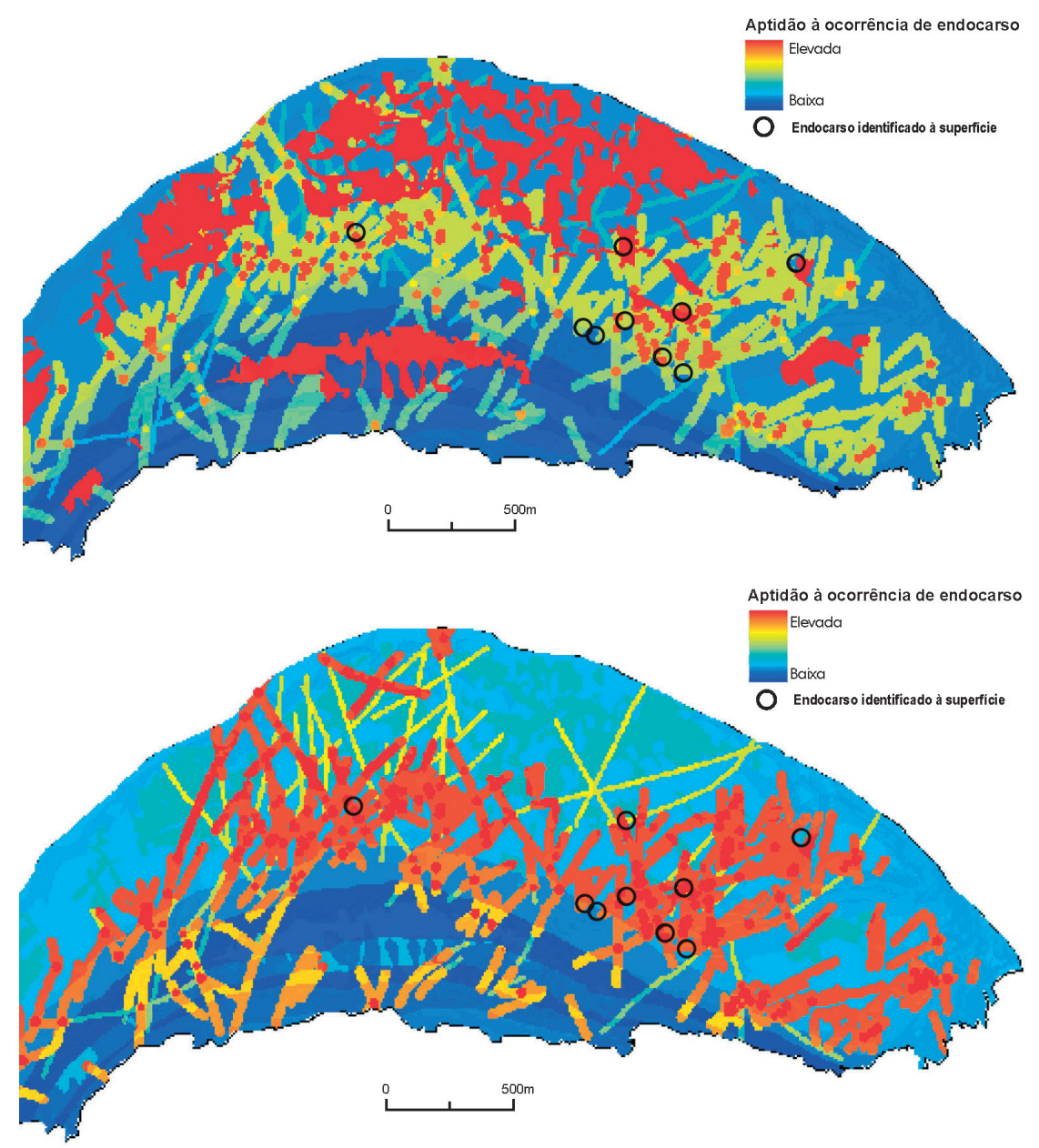

Fig. 6 - Aptidão à ocorrência de endocarso na área piloto para: a) sequência I: dissolução; intersecções; fracturação; litologia (1) ${ }^{3}$; litologia $(2)^{4}$; declive e b) sequência II: intersecções; fracturação; litologia $(1)^{3}$; dissolução; litologia $(2)^{4}$; declive.

Fig. 6 - Local aptitude to the occurrence of endokarst: a) sequence I: carbonate solution; intersections; fractures; lithology (1); lithology (2); slope and b) sequence II: intersections; fractures; lithology (1) and carbonates solution; lithology (2); slope.

${ }^{4}$ Litologia (2) - Separação das modalidades médio baixo, médio e elevado. 


\section{Análise dos resultados}

A diferença entre os dois mapas de aptidão da figura 6 resulta de se considerar a dissolução como um factor discriminativo a priori (hierarquizando as áreas com ocorrência de dissolução) ou, por outro lado, de dar a máxima importância à ocorrência das intersecções entre fracturas e fracturas propriamente ditas, independentemente da ocorrência de dissolução. Estes dois modos de combinar os factores e analisar/validar os resultados constituem um exemplo de alguns dos muitos testes de ordenação dos factores que foram efectuados.

De forma a validar os resultados obtidos, foram posteriormente realizados, de forma não sistemática, trabalhos de campo que permitiram a identificação de estruturas cársicas de superfície (algares), eventualmente indicadoras de um endocarso desenvolvido. Na figura 8 localizam-se nove estruturas cársicas identificadas na região, assinaladas na imagem por um círculo (área de influência de 30 metros) e a matriz do modelo final correspondente aos $25 \%$ de área mais apta para a ocorrência e prospecção de endocarso. A intenção de fazer a comparação utilizando uma incerteza de 30 metros deve-se ao facto de, durante o período de levantamento de campo da informação, terem sido introduzidos erros na sua localização espacial devido aos seguintes condicionalismos: (i) o rigor do

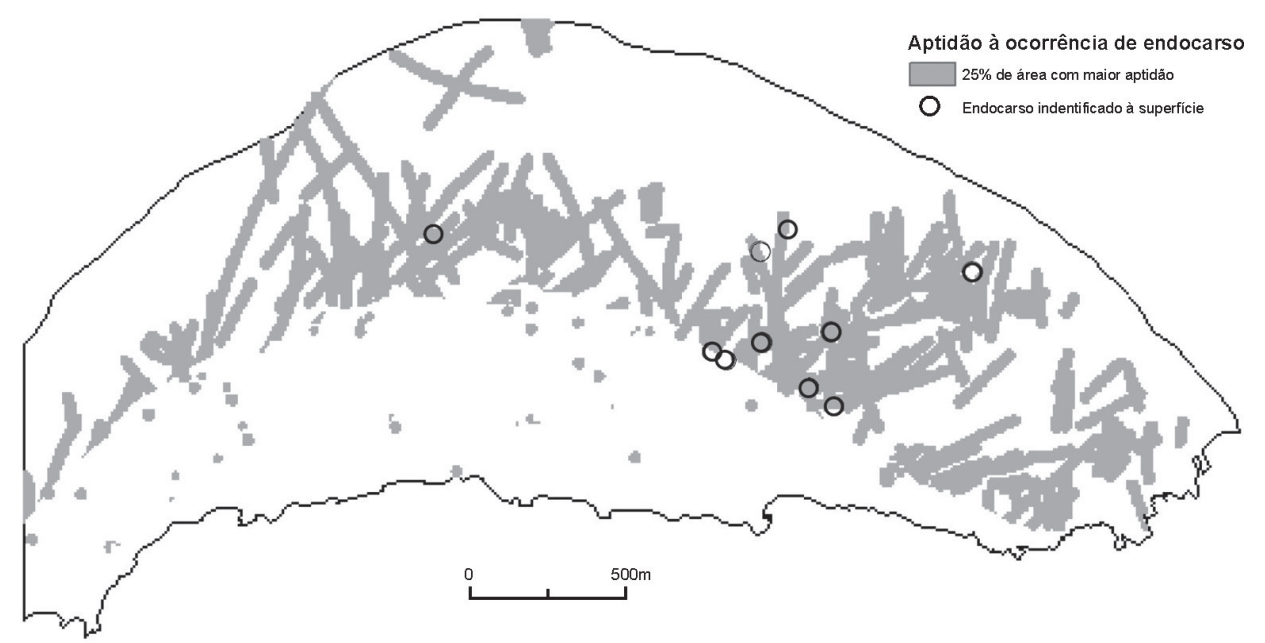

Fig. 7 - 25\% de área com maior aptidão à ocorrência de endocarso na área piloto estudada para a sequência da figura $6 \mathrm{~b}$.

Fig. $7-25 \%$ of the area showing the highest aptitude to the occurrence of endokarst within the training area for the figure $6 b$ sequence.

o equipamento GPS utilizado era de 5 a 15 metros na horizontal; (ii) o erro médio observado após georeferenciação das fotografias aéreas foi, igualmente, de 15 metros; (iii) a impossibilidade de ortorrectificação das fotografias aéreas por falta de disponibilidade de informação sobre o voo. 
Apesar da amostragem ser reduzida (nove locais), salienta-se que todas as cavidades inventariadas se encontram a menos de 30 metros de zonas classificadas como de maior aptidão (representando $25 \%$ da área total).

O modo de combinar a importância relativa dos factores, a maior ou menor extensão das áreas de influência relativamente à fracturação e intersecções, a própria escolha dos factores intervenientes e a subdivisão de um factor em várias modalidades, de forma a aplicar a discriminação em níveis distintos, são alguns exemplos dos graus de liberdade de aplicação do modelo, permitindo a calibração pericial dos resultados aos vários locais onde a litologia predominante seja do tipo carbonatado. Por outro lado, um exercício comparativo deste tipo constitui uma excelente oportunidade de compreensão dos parâmetros mais relevantes para a formação de endocarso.

\section{CONCLUSÕES}

A abordagem utilizada permitiu a construção de mapas indicando os locais com maior aptidão à ocorrência de estruturas endocársicas, com base em informação de superfície. Os factores mais relevantes para o desenvolvimento destas estruturas foram digitalizados e categorizados de forma a reflectir o grau de aptidão. Uma vez integrados no modelo SIG, permitiram definir os locais com maior potencial para a sua ocorrência.

Os resultados obtidos pela aplicação confirmam que a dispersão da aptidão para ocorrência de endocarso é fortemente controlada pela fracturação, de acordo com dados relativos a cavidades cársicas inventariadas durante este trabalho.

Este modelo desenvolvido em ambiente SIG estará apto a fornecer a entidades decisoras (tais como autarquias, associações espeleológicas, Parques Naturais, entre outras) uma ferramenta importante para o processo de prospecção de áreas de interesse espeleológico, assim que for possível elaborar a cartografia de carácter sistemático referida. Finalmente é importante referir que esta aplicação apresenta potencialidades para utilização em qualquer outra área geográfica desde que, para essa área, existam ou seja possível produzir os temas da fracturação e litologia. Naturalmente, quanto mais informação houver e quanto melhor a sua qualidade, melhor será o resultado da aplicação deste método.

\section{BIBLIOGRAFIA}

BLAIR, R. W. (1986) - Karst landforms and lakes, in Geomorphology from Space. Versão online: http:// daac.gsfc.nasa.gov/DAAC_DOCS/geomorphology/GEO_HOME_PAGE.html.

Corbel, J. (1959) - Les karsts du Yucatan et de Florida. Bull. Assoc. Geograph., France. 
CviJic, J. (1957) - Underground hidrology and morphological evolution of Karst. Sp. Ed. Serbian Geographical Society, Beograd, Vol. 35.

Fetter, C. W. (1980) - Applied hydrogeology. Book News, Inc., Portland, 691 p.

Ford, D. C. (1976) - Threshold and limit effects in karst geomorphology. In Coates, D. R and ViteK, J. D. - Thresholds in Geomorphology. Dowdm \& Culum, Inc., London: 345-362.

Manuppella, G.; Antunes, M. T.; Pais, J.; Ramalho, M. M. e Rey, J. (1994) - Carta Geológica de Portugal na escala de 1:50 000, Folha 38-B, IGM, Lisboa.

Manuppella, G.; Antunes, M. T.; Pais, J.; Ramalho, M. M. e Rey, J. ( (1999) - Notícia explicativa da Folha 38-B da Carta Geológica de Portugal na escala de 1:50 000, IGM, LIsboa.

PaRIzeK, R. R. (1976) - On the nature and significance of fracture traces and lineaments in carbonates and other terranes. Karst Hydrology and Water Resources (Proc. U.S. - Yugoslavian Symp.), Vol. 1, Fat Collins: 47-108.

Roglic, J. (1972) - Historical review of morphological concepts. In HeraK, M. and Springfield, T. (eds.) - Karst - Important Karst Regions of the Northern Hemisphere, Elsevier Pub. Co., The Netherlands: 1-18.

Smith, D. I. and Atrinson, T. C. (1976) - Process, landforms and climate in limestone regions, Geomorphology and Climate. Edited by E. Derbyshire. Wiley and Sons, Inc., New York: 367-409.

SweEting, M. M. (1972) - Karst Landforms. Macmillan, London.

Yevjevich, V. (1980) - Karst Water Research Needs. Water Resources Publications, Chelsea: 266 p. 